\title{
Prevalência de sífilis em mulheres do sistema prisional de uma capital do nordeste brasileiro
}

\section{Syphilis prevalence among women in the prison system of a northeastern Brazilian capital}

\section{Telma Maria Evangelista de Araújo ${ }^{1}$, Augusto Cezar Antunes de Araujo Filho ${ }^{2}$, Karla Vivianne Araújo Feitosa ${ }^{3}$}

\footnotetext{
${ }^{1}$ Enfermeira, Doutora em Enfermagem em Saúde Coletiva. Professor Adjunto da Universidade Federal do Piauí (UFPI). Teresina, PI, Brasil. E-mail: telmaevangelista@gmail.com.

${ }^{2}$ Enfermeiro. Discente do Programa de Pós-Graduação em Enfermagem, nível Mestrado, da UFPI. Teresina, PI, Brasil. E-mail: araujoaugusto@ @otmail.com.

${ }^{3}$ Enfermeira. Discente do Programa de Pós-Graduação em Enfermagem, nível Mestrado, da UFPI. Teresina, PI, Brasil. E-mail: karlynhavivi@hotmail.com.
}

\section{RESUMO}

Esta pesquisa objetivou investigar a prevalência de sífilis e fatores associados em internas na penitenciária feminina de Teresina-PI, Brasil. Estudo de corte transversal, realizado em novembro de 2013. A população foi constituída pelas internas da referida penitenciária $(n=131)$. Os dados foram coletados por meio de um formulário contendo questões fechadas e mistas. A média de idade foi 33,1 anos, 60,3\% afirmaram não manter relacionamento estável e 93,1\% possuíam filhos. O consumo de álcool foi referido por $70,8 \%$, e o uso de drogas ilícitas por $56,2 \%$. Evidenciou-se que $38,5 \%$ das mulheres nunca utilizam camisinha nas relações sexuais e que 62,2\% não sabem como se dá a transmissão da sífilis. A alta prevalência da sífilis, 25,2\%, está estatisticamente associada à situação conjugal, uso de drogas ilícitas e consumo antes das relações sexuais, demonstrando que condições socioeconômicas desfavoráveis são importantes marcadores de risco e de vulnerabilidade para as DST.

Descritores: Sífilis; Prisões; Mulheres; Enfermagem em Saúde Comunitária.

\section{ABSTRACT}

The aim of this study was to investigate the prevalence of syphilis and associated factors in inmates of the women's prison of Teresina, Piauí, Brazil. This cross-sectional study was conducted in November 2013. The population consisted of inmates from the referred penitentiary $(n=131)$. Data were collected through a form composed of closed-ended and mixed questions. The mean age was 33.1 years, $60.3 \%$ affirmed not having a stable relationship, and $93.1 \%$ had children. Alcohol use was reported by $70.8 \%$, and the use of illicit drugs, by $56.2 \%$. It was evidenced that $38.5 \%$ of women never use a condom during sexual intercourse, and that $62.2 \%$ do not know how the transmission of syphilis happens. The high prevalence of syphilis, $25.2 \%$, is statistically associated with marital status, illicit drug use and their consumption before sex, demonstrating that unfavorable socioeconomic conditions are important risk and vulnerability factors to sexually transmitted diseases.

Descriptors: Syphilis; Prisons; Women; Community Health Nursing. 


\section{INTRODUÇÃO}

O sistema prisional é considerado um problema de saúde pública no mundo, pois nos presídios existem condições que favorecem a propagação de doenças sexualmente transmissíveis (DST), uma vez que estes estabelecimentos apresentam condições relacionadas à violência, ao espaço físico limitado e a assistência à saúde inadequada ou incompleta ${ }^{(1-2)}$.

Também é importante ressaltar que no ambiente prisional a heterogeneidade dos indivíduos confinados, proporciona uma maior exposição aos riscos físicos e psicológicos $^{(3)}$. Diante disso, observa-se que as taxas de HIV e DST entre presos condenados são significativamente maiores que aquelas entre a população em geral, devido aos comportamentos de risco antes e durante o cárcere. A população carcerária mostra uma alta incidência de comportamentos de risco, que pode contribuir para uma maior propagação de infecções, especialmente no que diz respeito ao uso indevido de drogas injetáveis, tornando essa população mais exposta ao risco de adquirir infecções transmitidas pelo sangue ${ }^{(4-}$ 5). Além disso, outros estilos de vida e comportamentos de alto risco, também são observados, como: sexo desprotegido, múltiplos parceiros sexuais, homossexualidade e uso de tatuagem ${ }^{(6)}$.

A participação da mulher, no cenário prisional brasileiro, é significativamente baixa. Entretanto, devido a sua natureza, ela tende a sofrer mais intensamente ao cárcere, sendo, assim, mais vulnerável à aquisição de agravos à saúde, tanto no aspecto físico como no psicológico. Vários fatores cooperam para essa susceptibilidade, como: as características biológicas, as relações desiguais entre homens e mulheres, a ausência de oportunidade de falar sobre sexualidade e de conhecer seu corpo, a dificuldade em negociar o sexo com proteção, o não uso do preservativo, as condições socioeconômicas e culturais, as quais podem limitar o acesso aos serviços de saúde, a carência de ações e de informações adequadas com relação à prevenção das $\mathrm{DST}^{(3,7)}$.
Frente aos problemas expostos se faz necessário dispensar uma maior atenção à promoção da saúde da mulher privada de liberdade, tanto pelos maiores riscos presentes no ambiente prisional, como, também, pela deficiência de ações preventivas oferecidas no sistema de saúde carcerário ${ }^{(3)}$. Além disso, os serviços de saúde nas prisões, quando existem, são principalmente fora do sistema prisional, geralmente com capacidade insuficiente, caracterizada pela falta de pessoal e de outros recursos $^{(5)}$.

O acesso à saúde pela mulher privada de liberdade é um direito civil dificilmente observado no ambiente prisional brasileiro, o qual poderia ser melhorado por meio da promoção da saúde, privilegiando-se medidas preventivas e de promoção, que busquem transformar fatores que põem a coletividade em situação de iniquidade e vulnerabilidade ${ }^{(2-3)}$. Nesta perspectiva, o Governo Federal criou, em 2003, o Plano Nacional de Saúde no Sistema Penitenciário (PNSSP), que objetiva garantir o acesso à saúde pelas pessoas privadas de liberdade, oferecendo ações e serviços de atenção básica dentro das unidades prisionais ${ }^{(7)}$.

No estado do Piauí a situação de vulnerabilidade das mulheres internas em presídios, guarda muita semelhança com a existente em âmbito nacional e internacional. Destaca-se que ainda não há estudos suficientes, que determinem a real prevalência da sífilis no sexo feminino, dentro do sistema prisional. Desse modo, faz-se necessário o desenvolvimento de pesquisas voltadas para os agravos à saúde desse grupo de mulheres em desvantagem social, as quais precisam ter acesso igualitário aos serviços de saúde. Esta investigação poderá fornecer subsídios à realização de novas estratégias de promoção da saúde e prevenção de doenças sexualmente transmissíveis, de forma diferenciada e em conformidade com as singularidades do grupo em questão.

Frente ao exposto, este estudo objetivou investigar a prevalência da sífilis e fatores associados em internas da penitenciária feminina de Teresina/PI. 


\section{METODOLOGIA}

Estudo de corte transversal, que foi desenvolvido por meio de inquérito e pesquisa de marcador sorológico, com coleta de dados realizada em uma penitenciária feminina em uma capital do nordeste brasileiro, no mês de novembro de 2013. A população foi constituída pelo universo de internas da citada penitenciária $(n=131)$.

$\mathrm{Na}$ realização deste estudo foram respeitados os preceitos éticos legais baseados na Resolução do Conselho Nacional de Saúde (CNS) no 466/12. O projeto foi autorizado pela Secretaria de Justiça e aprovado pelo Comitê de Ética em Pesquisa da Universidade Federal do Piauí (CAAE: 17610613.4.0000.5214).

A execução aconteceu em duas etapas, sendo que na primeira realizaram-se entrevistas por meio da utilização de formulário contendo questões fechadas e mistas, referentes às características sociodemográficas, ao uso de álcool e outras drogas, à exposição parenteral, às práticas sexuais e às informaçãoes sobre DST/AIDS e, na segunda, foram realizados os testes rápidos pelo método da imunocromatografia para detecção de anticorpos da Sífilis (treponêmico), utilizando os testes TR DPP ${ }^{\circledR}$ Sífilis Bio- Manguinhos (Instituto de Tecnologia em imunobiológicos, Fundação Oswaldo Cruz, Rio de Janeiro, Brasil) e Rapid Check $^{\circledR}$ Sífilis (Núcleo de Doenças infecciosas, Universidade Federal do Espírito Santo, Vitória, Brasil). Os testes foram realizados por profissionais da Secretaria de Estado de Saúde do Piauí em conjunto com os autores, todos devidamente habilitados e qualificados pelo Ministério da Saúde, em treinamento próprio para execução e interpretação dos testes supracitados. Ressalta-se que todos os procedimentos para a aplicação destes testes seguiram as orientações e recomendações da Portaria Normativa no 3.242 de 30 de setembro de 2011.

Os dados foram coletados mediante o consentimento expresso das participantes, através do Termo de Consentimento Livre e Esclarecido (TCLE). Após isso, foram digitados no programa Microsoft Excel e importados para o programa Statistical Package for Social
Science (SPSS) versão 19.0, onde foram tabulados. A análise estatística utilizada foi a descritiva a partir dos percentuais das categorias de respostas das variáveis e explorados por meio das técnicas univariadas, bivariadas e multivariadas, considerando-se média, desvio padrão, intervalo de confiança de 95\%, mínimo e máximo. O uso do teste qui-quadrado com nível de significância $(p<0,05)$, foi utilizado para verificar as possíveis associações entre as variáveis. A partir da análise estatística bivariada foram selecionadas as variáveis que apresentaram nível de significância $p<0,25$, pelo teste qui-quadrado, para o modelo multivariado ${ }^{(8)}$. A análise multivariada foi realizada por meio de regressão binária logística, utilizando-se o odds ratio ajustado, com o respectivo IC95\% e nível de significância fixado em $p<0,05$. Foi examinada a ausência de multicolinearidade entre as variáveis selecionadas para a análise bivariada por meio do FIV (Variance-inflationfactor) e o ponto de corte para a existência de multicolinearidade adotado foi um FIV $\geq$ $4^{(9)}$.

\section{RESULTADOS}

A média de idade das detentas foi de 33,1 anos, com um intervalo de $18-68$, sendo que $58 \%$ declararam-se pardas/mestiças/mistas. Das internas, $64,9 \%$ não mantinham um relacionamento estável, incluindo-se solteiras, separadas e viúvas, entretanto, 95,4\% possuíam filhos, apresentando uma média de 2,5 filhos por detenta. Em relação à escolaridade, $87,8 \%$ frequentou a escola, com média de 7,1 anos de estudo. Das entrevistadas $82,4 \%$ possuíam um rendimento pessoal igual ou inferior a um salário mínimo. Um contingente de $85,5 \%$ não possuía plano de saúde e 72,5\% eram católicas. Das 131 mulheres, 90,8\% residiam no estado do Piauí, ao passo que somente $77,1 \%$ eram naturais desse estado (Tabela 1). 
Tabela 1: Perfil sociodemográfico e econômico das mulheres do estudo ( $n=131)$. Teresina, PI, Brasil, 2013.

\begin{tabular}{|c|c|c|c|c|c|}
\hline Variáveis & $n(\%)$ & $\overline{\bar{x}}$ & \pm & IC 95\% & Min-Max \\
\hline \multicolumn{6}{|l|}{ Procedência } \\
\hline Piauí & $101(77,1)$ & & & & \\
\hline Maranhão & $16(12,2)$ & & & & \\
\hline Outros & $14(13,7)$ & & & & \\
\hline \multicolumn{6}{|l|}{ Residência } \\
\hline Piauí & $119(90,8)$ & & & & \\
\hline Maranhão & $07(5,3)$ & & & & \\
\hline Outros & $05(3,9)$ & & & & \\
\hline Idade & & 33,1 & 11,9 & $31,1-35,2$ & $18-68$ \\
\hline Até 30 anos & $71(54,2)$ & & & & \\
\hline 31 e mais & $60(45,8)$ & & & & \\
\hline \multicolumn{6}{|l|}{ Cor da pele } \\
\hline Mista/Mestiça/Parda & $76(58,0)$ & & & & \\
\hline Branca & $24(18,3)$ & & & & \\
\hline Negra & $13(9,9)$ & & & & \\
\hline Outra & $10(7,6)$ & & & & \\
\hline Amarela & $08(6,1)$ & & & & \\
\hline \multicolumn{6}{|l|}{ Situação conjugal } \\
\hline Solteiro/separado/viúvo & $85(64,9)$ & & & & \\
\hline Casado/ União estável & $46(35,1)$ & & & & \\
\hline \multicolumn{6}{|l|}{ Possui Filhos } \\
\hline Sim & $125(95,4)$ & & & & \\
\hline Não & $06(4,6)$ & & & & \\
\hline Numero de filhos & & 2,5 & 1,7 & $2,2-2,8$ & $0-10$ \\
\hline Até 02 filhos & $77(58,8)$ & & & & \\
\hline 03 e mais & $54(41,2)$ & & & & \\
\hline \multicolumn{6}{|l|}{ Frequentou escola } \\
\hline Sim & $115(87,8)$ & & & & \\
\hline Não & $16(12,2)$ & & & & \\
\hline Anos de estudo & & 7,1 & 4,1 & $6,4-7,9$ & $0-17$ \\
\hline Até 5 & $48(36,6)$ & & & & \\
\hline 6 e mais & $83(63,4)$ & & & & \\
\hline Renda Pessoal (SM) & & 0,89 & 1,3 & $0,6-1,1$ & $0-08$ \\
\hline Até 1 & $108(82,4)$ & & & & \\
\hline 2 e mais & $23(17,6)$ & & & & \\
\hline Renda familiar (SM) & & 1,76 & 2,5 & $1,2-2,3$ & $0-20$ \\
\hline Até 1 & $78(59,5)$ & & & & \\
\hline 2 e mais & $53(40,5)$ & & & & \\
\hline \multicolumn{6}{|l|}{ Plano de saúde } \\
\hline Sim & $19(14,5)$ & & & & \\
\hline Não & $112(85,5)$ & & & & \\
\hline \multicolumn{6}{|l|}{ Religião } \\
\hline Católica & $95(72,5)$ & & & & \\
\hline Evangélica & $22(16,8)$ & & & & \\
\hline Nenhuma & $11(8,4)$ & & & & \\
\hline Outras & $03(2,3)$ & & & & \\
\hline
\end{tabular}

Legenda: $x=$ média, $\pm=$ Desvio padrão, IC95\%= intervalo de confiança, Min- Max= Mínima e máxima.

A maioria das participantes do estudo $(51,9 \%)$ referiu ter alguma informação sobre sífilis, entretanto, $60,3 \%$ não souberam informar como se dá a transmissão da doença. As mulheres que afirmaram saber como se dá esse processo destacaram as relações sexuais desprotegidas
$(89,8 \%)$ como o principal meio de contágio. Como principais fontes de informação as participantes elencaram os serviços de saúde, com $56,6 \%$, seguido da televisão, com 52,5\%. No que se refere à prevenção da sífilis $90,5 \%$ das detentas apontaram o preservativo como 
método mais eficaz. Das participantes, $95,4 \%$ respondeu que possui medo de contrair DST, e destacaram, como principais motivos, o fato de não ter cura $(25,6 \%)$, a morte (24\%) e o adoecimento (22,4\%) (Tabela 2$)$.
A prevalência de positividade do marcador sorológico para sífilis observada nesse grupo foi de $25,2 \%$ (IC95\%=16,3-28,3) (Tabela 3).

Tabela 2: Informações das internas sobre a sífilis ( $n=131)$. Teresina, PI, Brasil, 2013.

\begin{tabular}{|c|c|c|}
\hline Variáveis & $\mathbf{N}$ & $\%$ \\
\hline \multicolumn{3}{|l|}{ Informações sobre Sífilis } \\
\hline Sim & 68 & 51,9 \\
\hline Não & 63 & 48,1 \\
\hline \multicolumn{3}{|l|}{ Sabe como se dá a transmissão da sífilis } \\
\hline Sim & 44 & 33,6 \\
\hline Não & 79 & 60,3 \\
\hline Em parte & 08 & 6,1 \\
\hline \multicolumn{3}{|l|}{ Transmissão Sífilis* } \\
\hline Relações sexuais desprotegidas & 44 & 89,8 \\
\hline Sangue & 15 & 30,6 \\
\hline Transmissão vertical & 04 & 8,2 \\
\hline \multicolumn{3}{|l|}{ Fonte de informação sobre a transmissão da sífilis* } \\
\hline Serviço de saúde & 56 & 56,6 \\
\hline Televisão & 52 & 52,5 \\
\hline Outras & 22 & 22,2 \\
\hline Leitura & 18 & 18,2 \\
\hline \multicolumn{3}{|l|}{ Como prevenir-se da sífilis } \\
\hline Preservativo & 95 & 90,5 \\
\hline Outras & 05 & 4,9 \\
\hline Evitar relações sexuais & 05 & 4,8 \\
\hline \multicolumn{3}{|l|}{ Tem medo de pegar DST } \\
\hline Sim & 125 & 95,4 \\
\hline Não & 06 & 4,6 \\
\hline \multicolumn{3}{|l|}{ Motivo do medo } \\
\hline Não tem Cura/perigosa/grave/ruim & 32 & 25,6 \\
\hline Morrer & 30 & 24,0 \\
\hline Adoecer/comprometer a saúde & 28 & 22,4 \\
\hline Outras & 13 & 10,4 \\
\hline Tratamento difícil & 02 & 1,6 \\
\hline Porque na prisão todo mundo tem & 02 & 1,6 \\
\hline
\end{tabular}

Tabela 3: Prevalência de positividade do teste rápido para sífilis em internas da Penitenciária Feminina ( $n=131)$. Teresina, PI, Brasil, 2013.

\begin{tabular}{cccc}
\hline Variáveis & $\mathbf{n}$ & \% & \\
\hline Teste rápido para sífilis & & & \\
Positivo & 33 & 25,2 & $16,3-28,3$ \\
Negativo & 98 & 74,8 & $71,7-83,7$ \\
\hline
\end{tabular}

A análise bivariada evidenciou associação estatisticamente significativa entre o teste rápido de sífilis com as variáveis: situação conjugal $(p \leq 0,03)$, uso de drogas ilícitas $(p<0,01)$ e uso de drogas antes das relaçãos sexuais $(P<0,01)$ (Tabela 4$)$.

Na regressão logística múltipla as variáveis: situação conjugal, uso de drogas ilícitas e costuma usar drogas antes das relações sexuais mantiveram-se estatisticamente associadas à positividade do teste rápido para a sífilis $(p \leq 0,05)$. (Tabela 5$)$. 
Tabela 4: Associação dos dados sociodemográficos, uso de bebidas e outras drogas, práticas sexuais e informações sobre sífilis com o resultado do teste rápido ( $n=131)$. Teresina, PI, Brasil, 2013.

\begin{tabular}{|c|c|c|c|}
\hline \multirow{2}{*}{ Variáveis } & \multicolumn{2}{|c|}{ Teste Rápido de sífilis } & \\
\hline & Reagente & Não reagente & \\
\hline & $n(\%)$ & $n(\%)$ & p valor* \\
\hline Faixa etária & & & 0,72 \\
\hline Até 30 anos & $17(23,9)$ & $54(76,1)$ & \\
\hline 31 e mais & $16(26,7)$ & $44(73,3)$ & \\
\hline Renda pessoal & & & 0,09 \\
\hline Até 1 SM & $30(27,7)$ & $78(72,3)$ & \\
\hline $2 \mathrm{SM}$ e mais & $03(13,0)$ & $20(87,0)$ & \\
\hline Renda Familiar & & & 0,97 \\
\hline Até $1 \mathrm{SM}$ & $22(28,2)$ & $56(71,8)$ & \\
\hline $2 \mathrm{SM}$ e mais & $11(20,7)$ & $42(79,3)$ & \\
\hline Situação conjugal & & & 0,03 \\
\hline Solteiro & $25(31,6)$ & $54(68,4)$ & \\
\hline Casado & $08(15,4)$ & $44(84,6)$ & \\
\hline Tempo de estudo & & & 0,19 \\
\hline Até 05 anos & $15(31,9)$ & $32(68,1)$ & \\
\hline 6 anos e mais & $18(21,7)$ & $66(78,3)$ & \\
\hline Uso de bebida alcoólica & & & 0,25 \\
\hline Sim & $26(28,0)$ & $67(72,0)$ & \\
\hline Não & $07(18,4)$ & $31(81,6)$ & \\
\hline Uso de drogas ilícitas & & & $<0,01$ \\
\hline Sim & $26(35,1)$ & $48(64,9)$ & \\
\hline Não & $06(10,5)$ & $51(89,5)$ & \\
\hline Seleciona parceira(o) sexual & & & 0,23 \\
\hline Sim & $22(22,0)$ & $78(78,0)$ & \\
\hline Não & $11(35,5)$ & $20(64,5)$ & \\
\hline Usa camisinha & & & 0,19 \\
\hline Sempre & $11(36,6)$ & $19(63,4)$ & \\
\hline Nunca/Às vezes & $22(21,8)$ & $79(78,2)$ & \\
\hline Costuma usar bebida alcoólica antes das relações & & & 0,31 \\
\hline Sim & $17(24,3)$ & $53(75,7)$ & \\
\hline Não & $13(21,3)$ & $48(78,7)$ & \\
\hline Costuma usar drogas antes das relações & & & $<0,01$ \\
\hline Sim & $25(47,2)$ & $28(52,8)$ & \\
\hline Não & $08(10,3)$ & $70(89,7)$ & \\
\hline Informações sobre sífilis & & & 0,41 \\
\hline Sim & $15(22,1)$ & $53(77,9)$ & \\
\hline Não & $18(28,6)$ & $45(71,4)$ & \\
\hline
\end{tabular}


Tabela 5: Regressão logística múltipla dos fatores associados a prevalência de positividade do marcador sorológico para sífilis na população do estudo ( $n=131)$. Teresina, PI, Brasil, 2013.

\begin{tabular}{|c|c|c|c|c|}
\hline \multirow{3}{*}{ Variáveis } & \multicolumn{2}{|c|}{ Exame de sífilis } & \multirow{3}{*}{$\begin{array}{l}\text { O.R.a.** } \\
\text { p valor* }\end{array}$} & \multirow{3}{*}{ I.C. $95 \%$} \\
\hline & Reagente & Não reagente & & \\
\hline & $\mathrm{n}(\%)$ & n (\%) & & \\
\hline Renda pessoal & & & 0,81 & $-2,5-0,1$ \\
\hline Até $1 \mathrm{SM}$ & $26(26,0)$ & $74(74,0)$ & & \\
\hline 2 SM e mais $(*)$ & $01(6,7)$ & $14(93,3)$ & & \\
\hline Situação conjugal & & & 3,4 & $1,2-4,5$ \\
\hline Solteiro & $25(31,6)$ & $54(68,4)$ & & \\
\hline Casado $\left(^{*}\right)$ & $08(15,4)$ & $44(84,6)$ & & \\
\hline Tempo de estudo & & & 0,56 & $0,2-1,1$ \\
\hline Até 05 anos & $15(31,9)$ & $32(68,1)$ & & \\
\hline 6 anos e mais $(*)$ & $18(21,7)$ & $65(78,3)$ & & \\
\hline Uso de bebida alcoólica & & & 2,1 & $0,7-2,2$ \\
\hline Sim & $26(28,0)$ & $67(72,0)$ & & \\
\hline Não(*) & $07(18,4)$ & $31(81,6)$ & & \\
\hline Uso de drogas ilícitas & & & 4,4 & $2,7-5,6$ \\
\hline Sim & $26(35,6)$ & $47(64,4)$ & & \\
\hline Não(*) & $06(10,5)$ & $51(89,5)$ & & \\
\hline Seleciona parceira(o) sexual & & & 1,7 & $0,3-2,7$ \\
\hline $\operatorname{Sim}(*)$ & $21(22,1)$ & $74(77)$, & & \\
\hline Não & $09(33,3)$ & $18(66,7)$ & & \\
\hline Usa camisinha & & & 1,1 & $0,9-1,9$ \\
\hline Sempre $(*)$ & $11(36,6)$ & $19(63,4)$, & & \\
\hline Nunca/Às vezes & $22(21,8)$ & $79(78,2)$ & & \\
\hline Costuma usar drogas antes das relações sexuais & & & 3,6 & $1,9-4,6$ \\
\hline Sim & $22(51,2)$ & $21(48,8)$ & & \\
\hline Não(*) & $06(8,7)$ & $63(91,3)$ & & \\
\hline Tem medo de pegar DST & & & 1,8 & $0,5-2,2$ \\
\hline $\operatorname{Sim}(*)$ & $30(24,2)$ & $94(75,8)$ & & \\
\hline Não & $03(50,0)$ & $03(50,0)$ & & \\
\hline
\end{tabular}

*Categoria de Referência. O p valor foi obtido por Regressão Logística. Foram colocadas no modelo multivariado as variáveis com p $\leq 0,25$ no modelo bivariado. O nível de significância estatística foi fixado em $p \leq 0,05$.

\section{DISCUSSÃO}

Poucos estudos brasileiros analisaram a prevalência de sífilis em mulheres privadas de liberdade. Além disso, a comparação dos dados encontrados com os obtidos em outros estudos nacionais e internacionais não é simples, visto que existe uma diversidade de exames diagnósticos e que muitos desses estudos não contemplam o mesmo método diagnóstico e nem a DST incluída nesta investigação. Para viabilizar a discussão, os dados obtidos foram comparados, considerando-se a população feminina em geral e de outros grupos vulneráveis, e também são citados estudos que não utilizaram o mesmo método diagnóstico.

A utilização dos testes rápidos para o diagnóstico da sífilis tem se mostrado uma estratégia eficaz na implementação de novas medidas de abordagem para o diagnóstico rápido e preciso, assim como, no tratamento precoce durante a assistência à população em geral, principalmente na atenção às mulheres durante o prénatal e o parto. Assim como pode ser utilizado em situações específicas, nas quais exista dificuldade de acesso geográfico a serviços ou a insumos laboratoriais, como no contexto em que esta pesquisa foi desenvolvida ${ }^{(10-11)}$.

A alta prevalência detectada no presente estudo, (25,2\%; IC 95\%=16,3\% - 28,3\%), pode ser explicada devido à maioria da população não saber como se dá a transmissão da sífilis, assim como pode estar relacionada ao não uso ou à adesão irregular ao preservativo, relatado por uma elevada parcela da população estudada. Este 
achado apresenta-se em concordância com estudo internacional, realizado com a população carcerária feminina em Marrocos, cuja taxa de prevalência de sífilis foi de 23\%, sendo utilizados o Treponema Pallidum Haemagglutination Assay (TPHA) e o Venereal Disease Research Laboratory (VDRL) ${ }^{(12)}$.

Entretanto, observa-se que a prevalência deste estudo permanece elevada quando comparada com a população em geral, como os estudos realizados com puérperas no Brasil, 1,02\% (IC95\% 0,84-1,25) $)^{(13)}$ e em estudo desenvolvido em maternidades públicas de VitóriES, $0,4 \% \quad(I C 95 \% \quad 0,2-0,9)^{(10)}$. Ainda encontra-se em discrepância com estudos realizados com outros grupos vulneráveis, como em um desenvolvido com população em situação de rua de São Paulo, também utilizando testes rápidos, cuja prevalência foi de 7,0\% (IC95\% 2,6$9,4)^{(11)}$; com moradores de rua de São Paulo, 5,7\%, utilizando-se o VDRL ${ }^{(14)}$; em adolescentes do sistema correcional da grande Vitória, 7,8\% (IC95\% 2,6\%$12,8 \%)^{(15)}$; em prisões masculinas do Estado de São Paulo, $5,3 \% \quad(I C 95 \% \quad 3,5-7,6)^{(4)}$ e com presidiários de Pernambuco, 3,92\% (IC95\% 2,77-5,07\%) ${ }^{(16)}$.

Ao realizar-se a análise bivariada a presença do marcador sorológico reagente para sífilis, foi estatisticamente associada com a situação conjugal, uso de drogas ilícitas e uso de drogas antes das relações sexuais. No modelo multivariado as citadas variáveis mantiveram-se associadas. Tal achado pode ser explicado pelo risco de exposição à infecção ao longo da vida, por meio dos comportamentos de risco mencionados.

Algumas características peculiares de mulheres privadas de liberdade, descritas neste estudo e compatíveis com a literatura nacional e internacional, denota que elas possuem uma situação socioeconômica desfavorecida, apresentando baixa escolaridade e renda. Ademais, a associação encontrada com situação conjugal está intimamente relacionada à condição majoritária de mulheres solteiras, que possivelmente se relacionam com múltiplos parceiros, fato que configura maior vulnerabilidade às DST, principalmente, quando não fazem uso consistente do preservativo. Observou-se, em estudo realizado nos presídios de Gana, que o uso de drogas intravenosas, comportamentos sexuais de alto risco, e baixo nível socioeconômico resultaram na alta concentração de uma população de infectados com sífilis e outras $D_{S T}^{(17)}$.

Quando se trata de saúde, o uso de álcool e outras drogas podem gerar muitos prejuízos. Assim como a realidade detectada neste estudo, no qual a maioria das detentas relatou uso frequente de álcool e drogas, corroborando com uma pesquisa realizada em uma penitenciária feminina na cidade de São Paulo que evidenciou a maconha (61\%), a cocaína (47\%) e o crack (43\%), como as drogas mais utilizadas pelas detentas ${ }^{(18)}$. Tal fato intensifica a vulnerabilidade dessas mulheres, tendo em vista que isso desencadeia outros comportamentos de risco, como o compartilhamento de materiais infectantes para uso de drogas injetáveis e as práticas sexuais desprotegidas ${ }^{(15)}$.

O uso de substâncias psicoativas pode tornar as pessoas menos conscientes ou preocupadas com as DST, bem como com as práticas de rastreio convencionais dos serviços de saúde, que envolvem exame invasivo no trato genital, criando um distanciamento para com essa população ${ }^{(19)}$. Acrescenta-se o fato do consumo de drogas abranger questões amplas, que incluem desde atividades diretamente relacionadas ao seu uso, a troca de sexo por drogas e dinheiro e a uma maior liberdade sexual, incluindo múltiplos parceiros sexuais e práticas sexuais desprotegidas, que reflete no número elevado de casos novos de doenças nessa população ${ }^{(4,20)}$.

O não uso dos preservativos ou o uso irregular configura a realidade da penitenciária feminina deste estudo. Esse achado coaduna com outro estudo realizado no Ceará, em 2010, ${ }^{(21)}$ e evidencia a necessidade de estratégias de educação em saúde, que busquem refletir sobre as estratégias de promoção do conhecimento fornecidas pelas principais fontes referidas. Além disso, devem ser realizadas ações que promovam a saúde sexual e reprodutiva de presidiárias, as quais devem englobar a 
complexidade das peculiaridades vivenciadas pelas mulheres presidiárias e não apenas se restringir à realização de consultas ginecológicas e distribuição de preservativos $^{(3,21)}$.

Majoritariamente, as mulheres relataram alguma informação sobre sífilis, todavia grande parte delas não sabia como se dá a transmissão dessa doença. O cenário em que vivem constitui uma situação de vulnerabilidade, visto que o desconhecimento, as apresentações assintomáticas e a dificuldade de acesso às informações retarda o acesso aos serviços de saúde. A falta de informação sobre as várias DST, no que se refere aos sinais e sintomas e os modos de infecção são limitações reais que dificultam ainda mais esse acesso ${ }^{(15)}$.

Uma pesquisa internacional realizada na Arábia Saudita, com 261 mulheres constatou que mulheres reclusas possuem pouco conhecimento das DST, bem como sobre os comportamentos sexuais de risco ${ }^{(22)}$, fatos que dificultam a identificação das DST e causam a subestimação desses acometimentos ${ }^{(3)}$, o que tem gerado a necessidade da intensificação de campanhas com vistas ao aumento da conscientização sobre DST, principalmente entre a população carcerária, visto que constituem, em geral, populações de alto risco ${ }^{(22)}$.

Vieses de informação podem ter ocorrido em face das respostas autodeclaradas, especialmente relacionadas às questões consideradas estigmatizantes, como uso de drogas e parceria sexual, pois muitas participantes podem não ter relatado fidedignamente suas respostas, devido ao receio de que essas informações pudessem lhes proporcionar algum prejuízo em relação à sua situação jurídica e por receio de julgamentos morais sobre a sua conduta, embora tenham sido esclarecidos os objetivos da pesquisa e tenha sido deixado claro que essas informações eram sigilosas.

\section{CONCLUSÃO}

O grupo de detentas participante deste estudo foi constituído por mulheres que não mantinham relacionamento estável (64,9\%), que possuíam filhos
$(95,4 \%)$, apresentavam uma baixa escolaridade (média de 7,1 anos) e um baixo rendimento familiar (59,5\%). Apresentavam, ainda, comportamentos de riscos relacionados à sífilis, destacando-se o consumo de álcool $(71,8 \%)$, e outras drogas $(56,5 \%)$, enfatizando o uso antes das relações sexuais (44,3\% e 40,5\%, respectivamente), a não utilização de preservativo $(37,4 \%)$, além do baixo conhecimento sobre a transmissão da sífilis (60,3\%).

A alta prevalência de positividade do teste rápido para sífilis encontrada nos estudo $(25,2 \%)$ demonstrou que condições socioeconômicas desfavoráveis são importantes marcadores de risco e de vulnerabilidade para as DST, ou seja, a desigualdade social coloca as mulheres em situação de vulnerabilidade para diversos agravos de saúde, o que reflete a realidade estabelecida fora do sistema prisional.

Estudos de prevalência em populações carcerárias femininas são relevantes devido à heterogeneidade das mulheres reclusas e dos mais diversos comportamentos de risco que elas assumem. A questão de gênero tem uma significância maior nestes casos pelas condições inatas das mulheres e ainda pela limitação do acesso aos serviços de saúde e às informações adequadas com relação à prevenção das DST.

Diante disso, evidencia-se a necessidade de estratégias de saúde voltadas para a prevenção e diagnóstico precoce de DST tanto no contexto social em que essa população está inserida, quanto nos presídios, tendo em vista, que essa população está temporariamente reclusa, mas posteriormente será reinserida na comunidade de origem, e se não forem devidamente tratadas e educadas quanto à prevenção, casos novos continuarão acontecendo. Para tanto, é de extrema importância a implementação do Plano Nacional de Saúde no Sistema Penitenciário existente e traçar medidas condizentes com as características encontradas nas encarceradas. 


\section{REFERÊNCIAS}

1. Garaycochea M del C, Pino R, Chávez I, Portilla JL, Miraval $M L$, Arguedas $E$, et al. Infecciones de transmisión sexual en mujeres de un establecimiento penitenciario de Lima, Perú. Rev Peru Med Exp Salud Publica [Internet]. 2013 [acesso em: 31 dez. 2015];30(3):423-7. Disponível em:

http://www.scielo.org.pe/scielo.php?script=sci_arttext\&pid=S1 726-46342013000300008\&lng=pt\&nrm=iso\&tIng=es.

2. Agnolo CMD, Belentani LM, Jardim APS, Carvalho MDB, Pelloso SM. Perfil de mulheres privadas de liberdade no interior do Paraná. Rev. baiana saúde pública [Internet]. 2013 [acesso em: 31 dez. 2015];37(4):820-34. Disponível em: http://inseer.ibict.br/rbsp/index.php/rbsp/article/view/617.

3. Nicolau AIO, Ribeiro SG, Lessa PRA, Monte AS, Ferreira RCN, Pinheiro AKB. Retrato da realidade socioeconômica e sexual de mulheres presidiárias. Acta Paul Enferm [Internet]. 2012 [acesso em: 31 dez. 2015];25(3):386-92. Disponível em: http://dx.doi.org/10.1590/S0103-21002012000300011. 4. Maerrawi IE, Carvalho HB. Prevalence and risk factors associated with HIV infection, hepatitis and syphilis in a state prison of São Paulo. Int J STD AIDS [Internet]. 2015 [acesso em: 31 dez. 2015];26(2):120-7. Disponível em:

http://dx.doi.org/10.1177/0956462414531242.

5. Ravlija J, Vasilj I, Marijanović I, Vasilj M. Risk behaviour of prison inmates in relation to HIV/STI. Psychiatr Danub [Internet]. 2014 [acesso em: 31 dez. 2015];26 Supl. 2:395-401. Disponível em:

http://www.hdbp.org/psychiatria_danubina/pdf/dnb_vol26_s up2/dnb_vol26_sup2_395.pdf.

6. Nokhodian Z, Yazdani MR, Yaran M, Shoaei P, Mirian M, Ataei B, et al. Prevalence and Risk Factors of HIV, Syphilis, Hepatitis B and C Among Female Prisoners in Isfahan, Iran. Hepat Mon [Internet]. 2012 [acesso em: $31 \mathrm{dez}$.

2015];12(7):442-7. Disponível em:

http://dx.doi.org/10.5812\%2Fhepatmon.6144.

7. Ribeiro SG, Lessa PRA, Monte AS, Bernardo EBR, Nicolau AIO, Aquino PS, et al. Gynecologic and obstetric profile of state imprisoned females. Texto Context - Enferm [Internet]. 2013 [acesso em: $31 \mathrm{dez} .2015] ; 22(1): 13-21$. Disponível em: http://dx.doi.org/10.1590/S0104-07072013000100002.

8. Hosmer DW, Lemeshow, S. Applied logistic regression. $2^{\text {a }}$ ed. New York: Wiley; 2000.

9. StatNotes: Topics in Multivariate Analysis, from G. David Garson at North Carolina State University, Public Administration Program [Internet]. [acesso em: $31 \mathrm{dez}$. 2015]. Disponível em:

http://faculty.chass.ncsu.edu/garson/PA765/statnote.htm. 10. Miranda AE, Rosetti Filho E, Trindade CR, Gouvêa GM, Costa DM, Oliveira TG, et al. Prevalência de sífilis e HIV utilizando testes rápidos em parturientes atendidas nas maternidades públicas de Vitória, Estado do Espírito Santo. Rev Soc Bras Med Trop [Internet]. 2009 [acesso em: $31 \mathrm{dez}$. 2015];42(4):386-91. Disponível em: http://dx.doi.org/10.1590/S0037-86822009000400006. 11. Pinto VM, Tancredi MV, Alencar HDR De, Camolesi E, Holcman MM, Grecco JP, et al. Prevalence of Syphilis and associated factors in homeless people of Sao Paulo, Brazil, using a Rapid Test. Rev Bras Epidemiol [Internet]. 2014 [acesso em: 31 dez. 2015];17(2):341-54. Disponível em: http://dx.doi.org/10.1590/1809-4503201400020005ENG. 12. El Ghrari K, Terrab Z, Benchikhi H, Lakhdar H, Jroundi I, Bennani M. [Prevalence of syphilis and HIV infection in female prison population in Morocco]. East Mediterr Health J. 2007;13(4):774-9. [Article in French].

13. Domingues RMSM, Szwarcwald CL, Souza Junior PRB, Leal MC. Prevalence of syphilis in pregnancy and prenatal syphilis testing in Brazil: Birth in Brazil study. Rev Saude Publica [Internet]. 2014 [acesso em: 31 dez. 2015];48(5):766-74. Disponível em: http://dx.doi.org/10.1590/S00348910.2014048005114.

14. Brito VOC, Parra D, Facchini R, Buchalla CM. Infecção pelo HIV, hepatites B e C e sífilis em moradores de rua, São Paulo. Rev Saude Publica [Internet]. 2007 [acesso em: $31 \mathrm{dez}$. 2015];41 Supl. 2:47/56. Disponível em: http://dx.doi.org/10.1590/S0034-89102007000900009. 15. Miranda AE, Zago AM. Prevalência de infecção pelo HIV e Sífilis em sistema correcional para adolescentes. DST - J Bras Doenças Sex Transm [Internet]. 2001 [acesso em: $31 \mathrm{dez}$. 2015];13(4):35-39. Disponível em:

http://www.dst.uff.br/revista13-4-2001/c3.pdf.

16. Albuquerque ACC, Silva DM, Rabelo DCC, Lucena WAT, Lima PCS, Coelho MRCD, et al. Soroprevalência e fatores associados ao Vírus da Imunodeficiência Humana (HIV) e sífilis em presidiários do Estado de Pernambuco, Brasil. Cien Saude Colet [Internet]. 2014 [acesso em: 31 dez. 2015];19(7):2125-32. Disponível em: http://dx.doi.org/10.1590/141381232014197.08602013.

17. Adjei AA, Armah HB, Gbagbo F, Ampofo WK, Boamah I, Adu-Gyamfi C, et al. Correlates of HIV, HBV, HCV and syphilis infections among prison inmates and officers in Ghana: a national multicenter study. BMC Infect Dis [Internet]. 2008 [acesso em: $31 \mathrm{dez} .2015] ; 8(1): 33$. Available from: http://dx.doi.org/10.1186/1471-2334-8-33.

18. Strazza L, Azevedo RS, Carvalho HB. Prevenção do HIV/Aids em uma Penitenciária-modelo feminina de São Paulo - SP, Brasil. DST - J Bras Doenças Sex Transm [Internet]. 2006 [acesso em: 31 dez. 2015];18(4):235-40. Disponível em: http://www.dst.uff.br/revista18-42006/CAP3PrevencaodoHIVaidsemuma.pdf. 19. Bradshaw CS. Screening injecting drug users for sexually transmitted infections and blood borne viruses using street outreach and self collected sampling. Sex Transm Infect [Internet]. 2005 [acesso em: 31 dez. 2015];81(1):53-8. Disponível em: http://dx.doi.org/10.1136\%2Fsti.2004.009423. 20. Miranda AE, Merçon-de-Vargas PR, Viana MC. Saúde sexual e reprodutiva em penitenciária feminina, Espírito Santo, Brasil. Rev Saude Publica [Internet]. 2004 [acesso em: $31 \mathrm{dez}$. 2015];38(2):255-60. Disponível em: http://dx.doi.org/10.1590/s0034-89102004000200015. 21. Nicolau AIO, Ribeiro SG, Lessa PRA, Monte AS, Bernardo EBR, Pinheiro AKB. Conhecimento, atitude e prática do uso de preservativos por presidiárias: prevenção das DST/HIV no cenário prisional. Rev Esc Enferm USP [Internet]. 2012 [acesso 
em: 31 dez. 2015];46(3):711-9. Disponível em:

http://dx.doi.org/10.1590/S0080-62342012000300025.

22. Fageeh WM. Sexual behavior and knowledge of human immunodeficiency virus/aids and sexually transmitted infections among women inmates of Briman Prison, Jeddah, Saudi Arabia. BMC Infect Dis [Internet]. 2014 [acesso em: 31 dez. 2015];14(1):290. Disponível em:

http://dx.doi.org/10.1186/1471-2334-14-290.

Recebido: $12 / 03 / 2014$

Aceito: $18 / 03 / 2015$.

Publicado: 31/12/2015. 